\title{
Car License Plate Detection
}

\author{
Swapnil A. Pagore \\ Bhivarabai Sawant College of Engg \& Research, \\ Narhe, Pune, India.
}

\author{
M.S. Deshpande \\ Bhivarabai Sawant College of Engg \& Research, \\ Narhe, Pune, India.
}

\begin{abstract}
This paper deals with car license plate detection (CLPD) system in order to identify vehicles by capturing their car license plates (CLP). Car license plate detection (CLPDS) is an emerging area of research due to various applications such as prevention of crime, electronic toll system, intelligent traffic control system etc. In the proposed system, after converting the color input image into grayscale, an adaptive thresholding is used to obtain the binary image. Then the unwanted lines are removed through an unwanted-line elimination algorithm (ULEA). Finally to detect the license plate, vertical edges are detected by Sobel operator. Experiments were carried out for detection of front view as well as rear view of license plates. The experimental evaluation is carried out for 60 images taken from roadside and parking lots. The proposed method yields 96.9\% detection accuracy.
\end{abstract}

\section{General Terms}

License plate detection, License plate recognition.

\section{Keywords}

Adaptive thresholding (AT), car-license-plate-detection (CLPD), License Plate Detection (LPD).

\section{INTRODUCTION}

License plate recognition (LPR) system is used to identify vehicle by capturing their car license plates (CLPs). Licenseplate-detection (LPD), character segmentation and character recognition are the three main parts of car license plate detection and recognition system (CLPDRS). A LPD is the most important part in the system because it affects the system's performance.

Vehicle tracking and identification is used in many applications like vehicle access control, crime investigation and prevention, boarder control etc. In many vehicles, tracking systems cameras are used and installed in front of police cars to identify vehicle.

In the literature, there are different LPD methods proposed by different researchers such as, morphological operation [2][12], edge extraction [4]-[5], combination of gradient features [6], salience features [7], a neural network for color [8] or grayscale classification [10], vector quantization [11] etc. Detection of license plate is a challenging problem because of varying ambient lighting conditions, interference characters, font size etc. Matas and Zimmermann [14] proposed an algorithm to detect LPs under various conditions. This algorithm used character regions as basis units of LPs, which make the algorithm quite robust to view- point and illumination. However, it could hardly highlight characters overlapping from the true LPs. To address above issues, the proposed algorithms makes use of adaptive thresholding, ULEA as well as Sobel operator for vertical edge detection. The reminder of this paper is organized as follows. Section 2 describes methodology of car license plate detection. Section
3 gives experimental results and discussion. Conclusion and future scope is mentioned in Section 4.

\section{CAR LICENSE PLATE DETECTION}

The input image is captured by digital camera which has resolution $352 \times 288$. The image is resized to $256 \times 256$. The color input image is converted to a gray scale image as shown in Fig 3(a) and Fig 3(b). Adaptive thresholding (AT) is applied on the image to constitute the binarized image. After that, the ULEA is applied to remove noise and to enhance the binarized image. Next, the vertical edges are extracted by using Sobel operator. The desired plate details are highlighted. The next step is candidate regions extraction. After that, plate region selection (PRS) selects and extracts the correct LP. Finally license plate is detected. The flowchart of the proposed method is shown in Fig 1. All these steps are discussed further in detail.

\subsection{Adaptive Thresholding}

In order to account for variation in illumination, adaptive thresholding is used. In adaptive thresholding different threshold value is computed for each pixel in the image. An AT process [9] is applied after colour input image is converted to grayscale. The grayscale image is converted into binary through adaptive thresholding. Binary image is shown in Fig. 2(a)

\subsection{Unwanted Line Elimination Algorithm (ULEA)}

In general, thresholding process produces many thin lines that do not belong to LP region. There are many long foreground lines and short random noise edges beside the LP region as shown in Fig. 2(b). These lines may interfere in LP location. The number and alphabets on number plates are always in vertical orientation; therefore horizontal lines can be removed from binary image. The elimination or removal of these unwanted lines is termed as "ULEA". In the experiments performed, width of character is assumed to be only 15 pixels and only 15 pixels or more lines are eliminated.. There are three cases in which unwanted line can be formed. In the first, the line is horizontal with an angle equal to $0^{\circ}$ as $(-)$.The line is inclined with an angle equal to $45^{\circ}$ as $(/)$ in second case. The line is inclined by an angle equal to $135^{\circ}$ as $(\backslash)$ in the third case. After eliminating these lines, the holes in binary images are filled using morphological opening with line as a structuring element. 


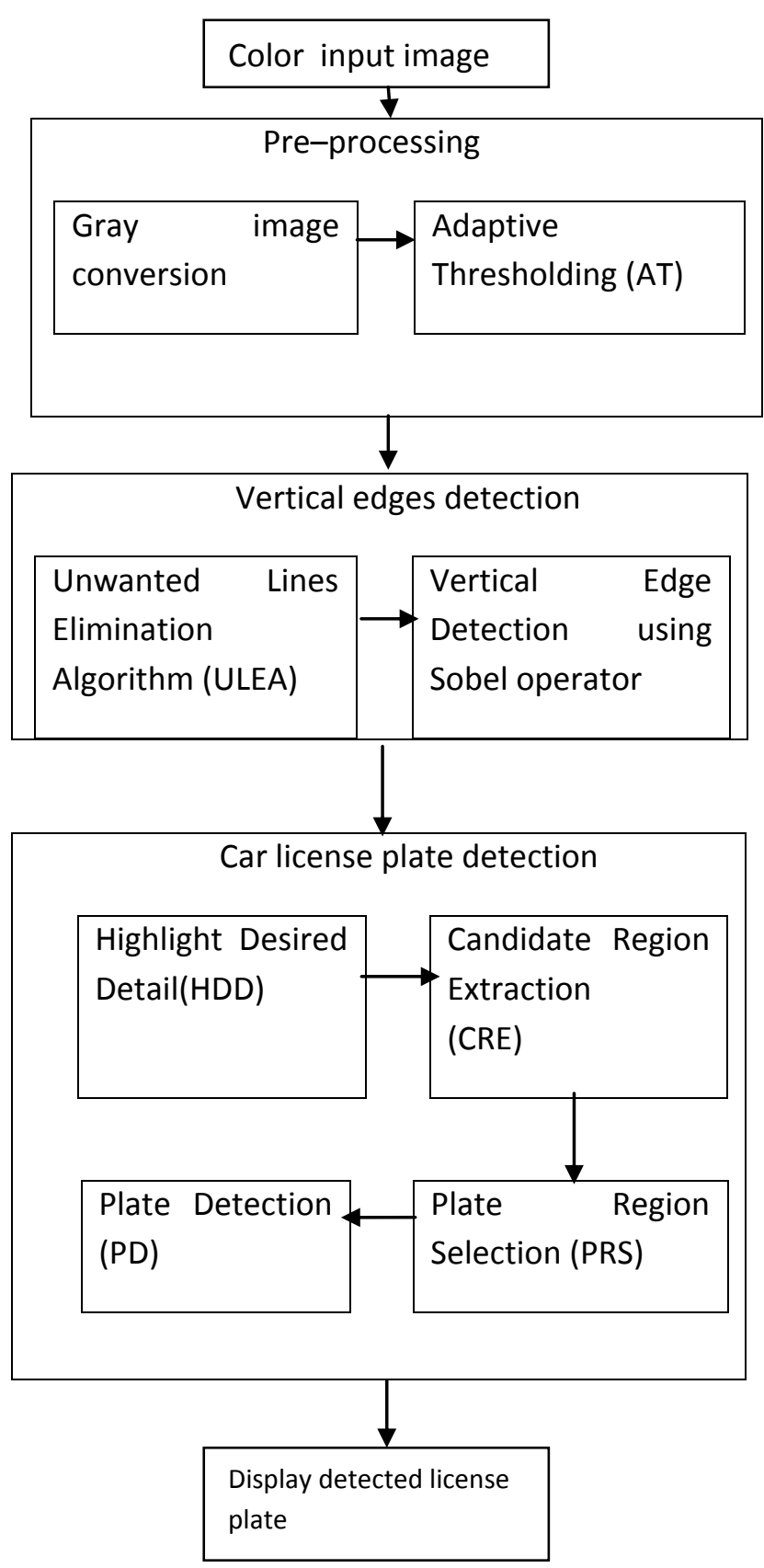

Fig 1: Flowchart of proposed system

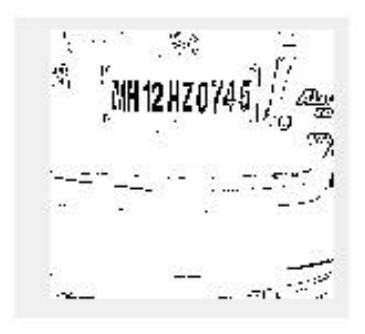

(a) Binary image

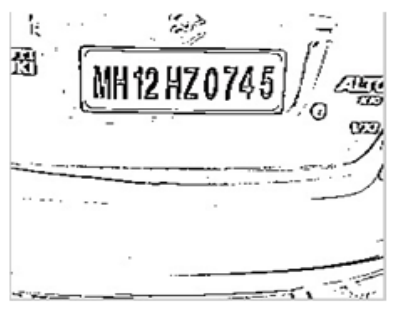

(b) ULEA
Fig 2: Output after applying ULEA on binary image

Many unwanted lines are removed after ULEA is applied. This kind of image is ready for a better segmentation process. The output of ULEA is shown in Fig. 2(b). The output clearly shows that many unwanted lines have been removed from binary image.

\subsection{Vertical Edge Detection using Sobel Operator}

Sobel operator is used for edge detection. After ULEA, it is required to localize number plate by considering all alphabets and numbers in vertical orientation. For this purpose, Sobel operator is used. There are many other vertical edges present however, select only those vertical lines which are closely placed and having small distance between them. The remaining vertical lines are removed. Fig.3(e) shows vertical edge detected output.

\subsection{Highlighting Desired Details}

After vertical edge detection, the next step is to highlight desired details like plate details and vertical edges in image. To highlight desired details two edges will be checked by drawing black horizontal lines connecting each two vertical edges. Horizontal distance $(h d)$ represents the length between two vertical edges of the single object. For removing long foreground and random edges that have not been eliminated earlier suitable $h d$ value is selected. While experimenting, selected $h d=30$. Those line having width less than 30 pixels are consider and remaining removed. Finally scanning process starts from left to right and from top to bottom. After scanning of all pixels, the region in which the correct LP exists is highlighted as shown in Fig. 3(f).

\subsection{Candidate Region Extraction}

Candidate region extraction process is divided into four steps such as count drawn lines per each row, divide image into multi-groups, count and store satisfied group indexes and boundaries, select boundaries of candidate regions.

1. Count drawn lines per each row: The lines drawn per each row will be counted and stored in matrix HwMnyLines $[a]$, where $a=0,1, \ldots$, height- 1 .

2. Divide the image into multi-group: To reduce the consumed time, gathering many rows as a group is used here. Dividing the image into multi-groups could be done using following:

how_mny_groups=height/C

Where how_mny_groups represents the total number of groups, height represents the total number of image rows, and $C$ represents the candidate region extraction (CRE) constant. The value of $C=10$ is selected. Here 10 rows are considered as one group.

1. Count and store satisfied group indexes and boundaries: Those group lines which are not part of the plate, are unsatisfied groups and eliminated. Each group is checked as; if it has at least 15 lines, then it is considered as part of LP regions. Finally, the total number of groups including parts of LP regions are counted and stored. The last step is to extract both upper and lower boundaries of each satisfied group by using its own index.

2. Select boundaries of candidate regions: This step draws the horizontal boundaries above and below each candidate regions. Fig. 3(g) shows the output of boundaries of drawing candidate regions. 


\subsection{Plate Region Selection}

Each column is checked one by one in candidate regions. The current column belongs to LP region if column blackness ratio exceeds $40 \%$. This column is replaced by vertical black line in result image as shown in Fig. 3(h). The condition for each column is checked, blckPix $\geq 0.4 \times$ colmnHght then current column is an element of LP region. Sometimes ratio of blackness to total length of candidate region is greater than $40 \%$. then some pixels of candidate will not be detected. The mathematical representation for selecting LP region is as follows:

$C_{\text {region }}=\left\{\begin{aligned} 0, & \text { blckPix } \geq \mathrm{P}_{\mathrm{RS}} \times \text { colmnHght } \\ 255, \text { otherwise } & \end{aligned}\right.$ (2)

Where, Cregion represents output value for current pixel of currently processed candidate region. If $C$ region $=0$, checked pixel as an element of LP region, otherwise consider it as background pixel. The PRS value is automatically determined. The detected license plate is as shown in Fig. 3(i).

\section{EXPERIMENTAL RESULTS AND DISCUSSION}

The database of 60 images is collected and classified into three groups. Group 1 consist of front view of the license plates. Group 2 consist of rear view of license plates. Group 3 consist of shadow appears on license plates. Table 1 shows experimental condition. Table 2 shows the detection accuracy. Table 2 shows that, the detection rate of G3 is lower than G1 and $\mathrm{G} 2$ due to complexity existing in images. The averaged detection rate of license plate is $96.9 \%$.

Table 1. Experimental conditions

\begin{tabular}{|c|c|}
\hline Environment & Outside \\
\hline Weather & Sunny \\
\hline Capturing times & $8 \mathrm{am}-6 \mathrm{pm}$ \\
\hline Total number of plates & 60 \\
\hline Camera distance to LP & $1 \mathrm{~m}$ to $2 \mathrm{~m}$ \\
\hline License plate design & Single row in LP \\
\hline
\end{tabular}

Table 2. Detection accuracy

\begin{tabular}{|c|c|}
\hline Group & Detection rate \\
\hline G1 & $97.5 \%$ \\
\hline G2 & $100 \%$ \\
\hline G3 & $93.3 \%$ \\
\hline Averaged detection rate & $96.9 \%$ \\
\hline
\end{tabular}

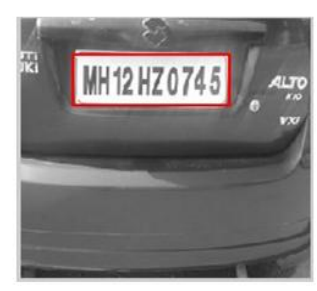

(a)

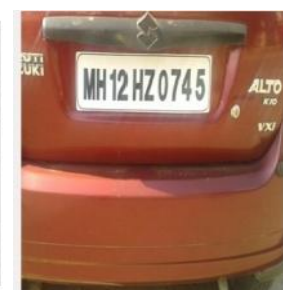

(b)

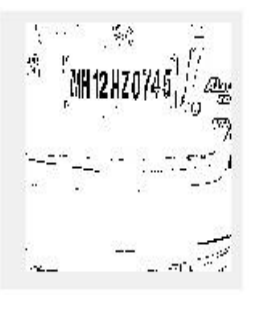

(a)

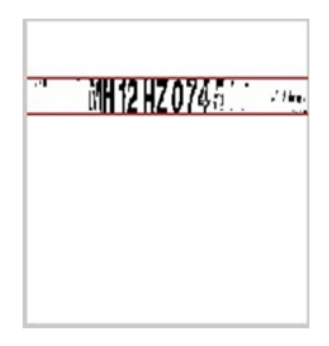

(e)

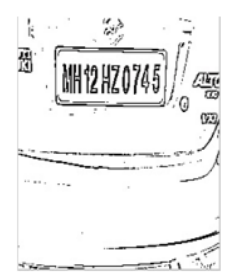

(d)

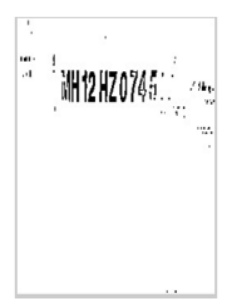

(f)

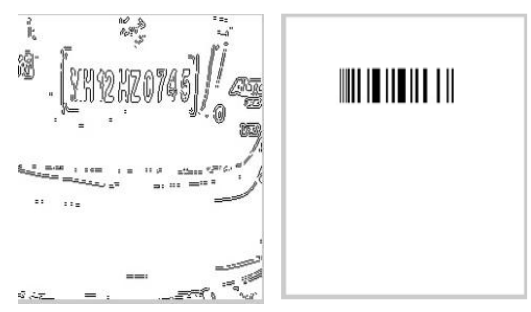

(e)

(f)

Fig. 3 (a) Original image (b) Grayscale image (c) Binary image (d) ULEA (e) Vertical edge detected output (f) HDD output (g) Candidate region (h) PRS output (i) LPD and LP extraction.

\section{CONCLUSION AND FUTURE SCOPE}

The main goal of this paper is to design robust LP detection system. The performance of the proposed algorithm is evaluated for 60 images captured using a digital camera from different location in different environment conditions. The averaged detection rate obtained is $96.9 \%$. CLPD system still has some challenges as varying lighting condition, tilt, varied size and design of LP script on the number plate and skew in image etc.

\section{REFERENCES}

[1] Abbas M. Al-Ghaili, Syamsiah Mashohor, Abdul Rahman Ramli, and Alyani Ismail "Vertical edge based car license plate detection method" IEEE transansation 
on vehicular technology, vol. 62, no. 1, pp. 2638 , January. 2013

[2] J.-W. Hsieh. S.-H. Yu, and Y.S. Chen, “ Morphologybased license plate detection from complex scenes," in proc. $16^{\text {th }}$ int. conf. pattern Recognit., Quebec city, QC, Canada, 2002, pp.176-179.

[3] F. Martin, o. Martin, M. Garcia, and J. L. Alba, "New methods for automatic reading of VLP's (Vehicle License Plates)," in proc. LASTED Int. Conf. signal Process Pattern Recognit, Appl., Heraklion, Greece, 2002, pp. 126-131.

[4] S.-H. Le, Y.-S. Seok, and E.-J. Lee, "Multi-national integrated car-license plate recognition system using geometrical feature and hybrid pattern vector," in proc. Int. Tech. Conf. Circuits Syst. Comput. Commun., Phuket, Thailand, 2002, pp. 1256-1259.

[5] J.-M. Guo and Y.-F. Liu, "License plate localization and character segmentation with feedback self-learning and hybrid binarization techniques," IEEE Trans. Veh. Technol., vol. 57, no. 3, pp. 1417-1424, May 2008.

[6] S. Kim, D. Kim, Y. Ryu, and G. Kim, "A robust licenseplate extraction method under complex image conditions," in Proc. 16th Int. Conf. Pattern Recognit., Quebec City, QC, Canada, 2002, pp. 216-219.

[7] Z.-X. Chen, Y.-L. Cheng, F.-L. Chang, and G.-Y. Wang, "Automatic license-plate location and recognition based on feature salience," IEEE Trans. Veh. Technol., vol. 58, no. 7, pp. 3781-3785, Sep. 2009.

[8] E. R. Lee, K. K. Pyeoung, and J. K. Hang, "Automatic recognition of a car license plate using color image processing," in Proc. IEEE Int. Conf. Image Process., 1994, pp. 301-305.

[9] D. Bradley and G. Roth, "Adaptive thresholding using integral image,' J. Graph. Tools, vol. 12, no. 2, pp. 1321, Jun. 2007

[10] H. Caner, H. S. Gecim, and A. Z. Alkar, "Efficient embedded neural network-based license plate recognition system," IEEE Trans. Veh. Technol., vol. 57, no. 5, pp. 2675-2683, Sep. 2008.

[11] S. Rovetta and R. Zunino, "License-plate localization by using vector quantization," in Proc. Int. Conf. Acous. Speech, Signal Process., 1999, pp. 1113-1116.

[12] L. S. Davis, "A survey of edge detection techniques," J.Comput. Graph. Image Process., vol. 4, no. 3, pp. 248 270, Sep. 1975.

[13] J. K. Aggarwal, R. O. Duda, and A. Rosenfeld, Computer Methods in Image Analysis. New York: IEEE Press, 1977

[14] J. Matas and K. Zimmermann, "Unconstrained license plate and text localization and recognition," in Proc. IEEE Int. Conf. Intell. Transp. Syst., Vienna, Austria, 2005 , pp. 572-577. 